\title{
TOPOLOGIES ON THE SYMMETRIC INVERSE SEMIGROUP
}

\author{
J. PÉREZ AND C. UZCÁTEGUI
}

\begin{abstract}
The symmetric inverse semigroup $I(X)$ on a set $X$ is the collection of all partial bijections between subsets of $X$ with composition as the algebraic operation. We study a minimal Hausdorff inverse semigroup topologies on $I(X)$. When $X$ is countable, we show some Polish semigroup topologies on $I(X)$.
\end{abstract}

\section{INTRODUCTION}

The symmetric inverse semigroup $I(X)$ on a set $X$ is the collection of all partial bijections between subsets of $X$ with composition as the algebraic operation. Among inverse semigroups, $I(X)$ plays a role analogous to that played by the symmetric group $S_{\infty}(X)$ for groups: every inverse semigroup $S$ is isomorphic to a subsemigroup of $I(S)$. In this paper we study some semigroup topologies on $I(X)$. In the case of a countable $X$, the topologies on $I(X)$ can be found Polish (i.e. completely metrizable and separable).

The symmetric group $S_{\infty}(X)$ is a subsemigroup of $X^{X}$ and also of $I(X)$. The usual topology of $S_{\infty}(X)$ is the one it inherits from the product topology on $X^{X}$. We define a topology $\tau_{p p}$ on $I(X)$, we call it the partial product topology, which also induces in $S_{\infty}(X)$ its usual product topology. It turns out that $\tau_{p p}$ is the minimal inverse semigroup Hausdorff topology on $I(X)$. We present a characterization of $\tau_{p p}$ analogous to that well known fact that the product topology is the smallest topology making all projections continuous. We show that there is an onto map $\pi: S_{\infty}(Y) \rightarrow I(X)$ such that $\tau_{p p}$ is the quotient topology given by $\pi$. Moreover, $\tau_{p p}$ is the unique inverse semigroup Hausdorff topology on $I(X)$ with respect to which $\pi$ is continuous. The topology $\tau_{p p}$ was independently studied in [1, 2. Some of the results we present are coming from [7.

Recently there has been a lot of interest about groups and semigroups which admits a unique Polish group (semigroup) topology. A theorem of Kallman [4 says that $S_{\infty}(\mathbb{N})$ has a unique Polish group topology, namely the product topology (see [3, Exercise 2.3.9]). The same happens with the group of homeomorphisms of the Cantor space as shown by Rosendal and Solecki [8]. Mesyan et al. [6] showed that the product topology is the unique Polish semigroup topology on $\mathbb{N}^{\mathbb{N}}$. More recently, Elliott et. al made a very extensive study of Polish semigroup topologies [1, 2]. In particular, they showed that there is a unique Polish inverse semigroup topology on $I(\mathbb{N})$, namely $\tau_{p p}$. Their proof is based on a general criteria for getting automatic continuity for $I(\mathbb{N})$ using the fact that $S_{\infty}(\mathbb{N})$ admits an unique Polish group topology. Our approach is quite different but we obtained a weaker result, since it needs some extra condition (see Theorem [6.6).

\section{Preliminaries}

A semigroup is a non-empty set $S$ together an associative binary operation $\circ$. To simplify the notation we sometimes write $s t$ in placed of $s \circ t$. Let $S$ be a semigroup. We say that $S$ is a regular if for all $s \in S$, there is $t \in S$ such that $s t s=s$ and $t s t=t$. In this case we say that $t$ is an inverse

Date: December 8, 2020.

2020 Mathematics Subject Classification. Primary 24A15, 03E15; Secondary 54H15.

Key words and phrases. Inverse semigroup, Topological semigroup, Polish semigroup. 
of $s$. If each element have a unique inverse we call $S$ an inverse semigroup. We denote by $s^{*}$ the inverse of $s$.

Let $S$ be a semigroup and $\tau$ a topology on $S$. If the multiplication $S \times S \rightarrow S$ is continuous, we call $S$ a topological semigroup. An inverse semigroup $S$ is called topological, if it is a topological semigroup and the function $i: S \rightarrow S, s \rightarrow s^{*}$ is continuous.

Proposition 2.1. Let $S$ be a inverse semigroup and $\tau$ a topology on $S$ such that $(S, \tau)$ is a Hausdorff topological semigroup. Suppose $\left(s_{n}\right)_{n}$ is a sequence on $S$ such that $s_{n} \rightarrow s$ and $s_{n}^{*} \rightarrow t$. Then $t=s^{*}$.

Proof. Notice that $s_{n}=s_{n} s_{n}^{*} s_{n}$ and $s_{n}^{*}=s_{n}^{*} s_{n} s_{n}^{*}$ for all $n \in \mathbb{N}$. By the continuity of the semigroup operation, we have that $s_{n}=s_{n} s_{n}^{*} s_{n} \rightarrow s t s$ and $s_{n}^{*}=s_{n}^{*} s_{n} s_{n}^{*} \rightarrow t s t$. Thus $s=s t s$ and $t=t s t$, in other words, $t=s^{*}$.

Let $S$ be an inverse semigroup and $\rho$ a metric on $S$. Define another metric as follow

$$
\rho^{*}(s, t)=\rho\left(s^{*}, t^{*}\right) .
$$

Proposition 2.2. Let $S$ be a inverse semigroup and $\rho$ a metric on $S$.

(i) $i:\left(S, \tau_{\rho}\right) \rightarrow\left(S, \tau_{\rho^{*}}\right), s \mapsto s^{*}$, is an isometry. Moreover, if $\rho$ is complete, so is $\rho^{*}$.

(ii) Suppose $\left(S, \tau_{\rho}\right)$ is a topological semigroup, then $\left(S, \tau_{\rho^{*}}\right)$ is a topological semigroup.

Proof. (i) It is straightforward.

(ii) Define $g:\left(S, \rho^{*}\right) \times\left(S, \rho^{*}\right) \rightarrow S \times S$ such that $(s, t) \rightarrow(t, s)$. Note that $g$ is continuous function on $\tau_{\rho^{*}}$ and $*=i \circ * \circ(i \times i) \circ g$, therefore $*$ is a continuous function on $\tau_{\rho^{*}}$. And $\rho^{*}$ is complete since $\rho$ is complete and $i$ is an isometric homeomorphism by Proposition 2.2(i).

Theorem 2.3. Let $S$ be an inverse semigroup and $\rho$ be a complete metric on $S$ such that $\left(S, \tau_{\rho}\right)$ is a topological semigroup. Let $d=\rho+\rho^{*}$, then $d$ is a complete metric on $S$ and $\left(S, \tau_{d}\right)$ is a topological inverse semigroup.

Proof. Let us see that $d$ is complete. Let $\left(s_{n}\right)_{n}$ be a $d$-Cauchy sequence and $\epsilon>0$, then there is $N \in \mathbb{N}$ such that if $n, m>N, d\left(s_{n}, s_{m}\right)<\epsilon$. Thus $\rho\left(s_{n}, s_{m}\right)<\epsilon$ and $\rho^{*}\left(s_{n}, s_{m}\right)<\epsilon$ for all $n, m>N$. Since $\rho$ and $\rho^{*}$ are complete, there are $s, t \in S$ such that $s_{n} \stackrel{\rho}{\longrightarrow} s$ and $s_{n} \stackrel{\rho^{*}}{\longrightarrow} t$. By Proposition 2.1, $t=s$. It is easy to see that $s_{n} \stackrel{d}{\longrightarrow} s$.

Now we show that $\tau_{d}$ is an inverse semigroup topology. First we note that $i^{-1}\left(B_{d}(s, r)\right)=$ $B_{d}\left(s^{*}, r\right)$, thus $i$ is continuous. To see that the operation $\left(S, \tau_{d}\right) \times\left(S, \tau_{d}\right) \rightarrow\left(S, \tau_{d}\right)$ is continuous,

let $\left(x_{n}\right)_{n}$ and $\left(y_{n}\right)_{n}$ be sequences such that $x_{n} \stackrel{d}{\rightarrow} x$ and $y_{n} \stackrel{d}{\rightarrow} y$, we show that $x_{n} y_{n} \stackrel{d}{\rightarrow} x y$. Clearly

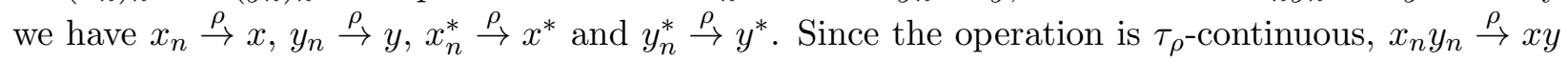
and $\left(x_{n} y_{n}\right)^{*} \stackrel{\rho}{\rightarrow}(x y)^{*}$, therefore $x_{n} y_{n} \stackrel{d}{\rightarrow} x y$.

\section{The PARTIAL PRODUCT TOPOLOGY}

The symmetric inverse semigroup on a set $X$ is defined as follows:

$$
I(X)=\{f: A \rightarrow B \mid A, B \subseteq X \text { and } f \text { is bijective }\} .
$$

For $f: A \rightarrow B$ in $I(X)$ we denote $A=\operatorname{dom}(f)$ and $B=\operatorname{im}(f)$. Let

$$
D_{x}=\{f \in I(X): x \in \operatorname{dom}(f)\}
$$

and $2^{X}$ denotes the power set of $X$. Let $S_{\infty}(X)$ be the symmetric group, that is, the collection of all bijection from $X$ to $X$. The following functions play an analogous role as the proyection functions for the product topology. 


$$
\begin{aligned}
& \operatorname{dom}: I(X) \rightarrow 2^{X}, f \mapsto \operatorname{dom}(f), \\
& \operatorname{im}: I(X) \rightarrow 2^{X}, f \mapsto \operatorname{im}(f), \\
& \operatorname{ev}_{x}: D_{x} \rightarrow X, f \mapsto f(x) \text { for all } x \in X .
\end{aligned}
$$

The operation on $I(X)$ is the usual composition, namely, given $f, g \in I(X)$, then $f \circ g$ is defined by letting $\operatorname{dom}(f \circ g)=g^{-1}(\operatorname{dom}(f) \cap \operatorname{im}(g))$ and if $x \in \operatorname{dom}(f \circ g)$ then $(f \circ g)(x)=f(g(x))$. The idempotents of $I(X)$ are the partial identities $1_{A}: A \rightarrow A, 1_{A}(x)=x$ for all $x \in A$ and $A \subseteq X$. Notice that $1_{\emptyset}$ is the empty function which also belongs to $I(X)$. For $x, y \in X$, let

$$
\begin{aligned}
v(x, y) & =\{f \in I(X) \mid x \in \operatorname{dom}(f) \text { and } f(x)=y\}, \\
w_{1}(x) & =\{f \in I(X) \mid x \notin \operatorname{dom}(f)\}, \\
w_{2}(y) & =\{f \in I(X) \mid y \notin \operatorname{im}(f)\} .
\end{aligned}
$$

It is clear that sets $v(x, y)$ are motivated by the usual subbase for the product topology on $X^{X}$. As we will see, these sets are tightly related to any $T_{1}$ semigroup topology on $I(X)$.

Lemma 3.1. Let $c: I(X) \times I(X) \rightarrow I(X)$ be given by $c(f, g)=f \circ g$ and $i: I(X) \rightarrow I(X)$ be given by $i(f)=f^{-1}$. Then, for all $x, y \in X$, we have

(i) $c^{-1}(v(x, y))=\bigcup_{z \in X}(v(z, y) \times v(x, z))$.

(ii) $c^{-1}\left(w_{1}(x)\right)=\left(I(X) \times w_{1}(x)\right) \cup \bigcup_{z \in X}\left(w_{1}(z) \times v(x, z)\right)$.

(iii) $c^{-1}\left(w_{2}(y)\right)=\left(w_{2}(y) \times(I(X)) \cup \bigcup_{z \in X}\left(v(z, y) \times w_{2}(z)\right)\right.$.

(iv) $i^{-1}(v(x, y))=v(y, x)$,

(v) $i^{-1}\left(w_{1}(x)\right)=w_{2}(x)$,

(vi) $i^{-1}\left(w_{2}(y)\right)=w_{1}(y)$.

Proof. It is straightforward.

Now we introduce some semigroup topologies on $I(X)$. Let $\tau_{0}$ be the topology generated by $\{v(x, y): x, y \in X\}, \tau_{1}$ be the topology generated by $\left\{v(x, y), w_{1}(x): x, y \in X\right\}, \tau_{2}$ be the topology generated by $\left\{v(x, y), w_{2}(y): x, y \in X\right\}$ and $\tau_{p p}$ be the topology generated by $\left\{v(x, y), w_{1}(x), w_{2}(y)\right.$ : $x, y \in X\}$. These topologies were independently defined in [1, 2] and show essentially the following.

Theorem 3.2. $\quad$ (i) $\left(I(X), \tau_{0}\right)$ is a $T_{0}$ topological semigroup but it is not $T_{1}$.

(ii) $\left(I(X), \tau_{1}\right)$ is a Hausdorff topological semigroup.

(iii) $\left(I(X), \tau_{2}\right)$ is a Hausdorff topological semigroup and $i:\left(I(X), \tau_{1}\right) \rightarrow\left(I(X), \tau_{2}\right)$ is a homeomorphism and an antisomorphism.

(iv) $\left(I(X), \tau_{p p}\right)$ is a Hausdorff topological inverse semigroup.

Proof. Let $\tau$ be any of the topologies mentioned in the hypothesis. From Lemma 3.1]it follows that $\tau$ is a semigroup topology on $I(X)$.

(i) It remains to show that the $\tau_{0}$ is $T_{0}$. In fact, let $f, g \in I(X)$ with $f \neq g$. There are several cases to consider, we treat only one, the others are analogous. Assume there is $x \in \operatorname{dom}(f) \cap \operatorname{dom}(g)$ such that $f(x) \neq g(x)$. Then $f \in v(x, f(x)))$ and $g \notin v(x, f(x))$. Notice that the only $\tau_{0}$-open set that contains $1_{\emptyset}$ is $I(X)$, therefore $\left(I(X), \tau_{0}\right)$ is not $T_{1}$.

(ii) It remains to show that $\tau_{1}$ is $T_{2}$. This is done easily by analyzing the following cases. Let $f \neq g$ in $I(X)$. (a) There is $x \in \operatorname{dom}(f) \triangle \operatorname{dom}(g)$ (b) There is $x \in \operatorname{dom}(f) \cap \operatorname{dom}(g)$ with $f(x) \neq g(x)$.

(iii) It follows from Lemma 3.1. (iv)It follows from Lemma 3.1.

We call $\tau_{p p}$ the partial product topology. The following result shows that $\tau_{p p}$ is minimal among all Hausdorff inverse semigroup topologies on $I(X)$. 
Theorem 3.3. Suppose $(I(X), \tau)$ is a $T_{1}$ topological semigroup. Then

(i) Each $v(x, y)$ is clopen and $w_{1}(y)$ and $w_{2}(y)$ are closed for all $y$.

(ii) Suppose $\tau$ is an inverse semigroup topology. The relation $\subseteq$ is closed on $I(X) \times I(X)$ iff every $w_{1}(x)$ is open.

(iii) If $\tau$ is an inverse semigroup Hausdorff topology, then $\tau_{p p} \subseteq \tau$.

Proof. (i) For each $x, y \in X$, let $u_{x, y} \in I(X)$ be such that $\operatorname{dom}\left(u_{x, y}\right)=\{x\}$ and $\operatorname{im}\left(u_{x, y}\right)=\{y\}$. Let $\varphi: I(X) \rightarrow I(X)$ given by $\varphi(h)=u_{y, x} \circ h \circ u_{y, x}$. Then $\varphi$ is continuous and

$$
I(X) \backslash v(x, y)=\varphi^{-1}\left(1_{\emptyset}\right) .
$$

Thus $v(x, y)$ is open. We also have that $v(x, y)=\varphi^{-1}\left(u_{y, x}\right)$. Thus $v(x, y)$ is also closed. To see that $w_{1}(x)$ and $w_{2}(y)$ are closed observe that $I(X) \backslash w_{1}(x)=\bigcup_{z \in X} v(x, z)$ and $I(X) \backslash w_{2}(y)=$ $\bigcup_{z \in X} v(z, y)$.

(ii) Suppose $\subseteq$ is closed. Then $f \notin w_{1}(x)$ iff $u_{x, x} \subseteq f^{-1} \circ f$. Thus the complement of $w_{1}(x)$ is closed. Conversely, $f \nsubseteq g$ iff for some $x, y \in X$ we have that $f \in v(x, y)$ and $g \in((I(X) \backslash v(x, y)) \cup$ $w_{1}(x)$. From this follows that the complement of $\subseteq$ is open.

(iii) Suppose that $\tau$ is an inverse semigroup Hausdorff topology on $I(X)$. From (i) we have that each $v(x, y)$ is $\tau$-open. To see that each $w_{1}(x)$ is $\tau$-open, by (ii), it suffices to show that $\subseteq$ is $\tau \times \tau$-closed. In fact, notice that $f \subseteq g$ iff $f=f \circ f^{-1} \circ g$. Since $\circ$ is $\tau$-continuous, then $\subseteq$ is closed. Finally, since $\tau$ is an inverse semigroup topology and each $w_{1}(x)$ is $\tau$-open, from Lemma 3.1 we conclude that each $w_{2}(y)$ is also $\tau$-open. Thus $\tau_{p p} \subseteq \tau$.

Corollary 3.4. $\left(I(X), \tau_{p p}\right)$ is a regular space.

Next we present a characterization of when the collection of idempotent of $I(X)$ is compact.

Theorem 3.5. Let $\tau$ be an inverse semigroup Hausdorff topology on $I(X)$. The map $A \mapsto 1_{A}$ from $2^{X}$ to $I(X)$ is continuous iff the collection of idempotents is compact.

Proof. The collection of idempotent of $I(X)$ is $J=\left\{1_{A}: A \subseteq X\right\}$. Thus if the map $A \mapsto 1_{A}$ is continuous, then the collection of idempotent is compact. Conversely, suppose $J$ is compact. By Theorem 3.8, the function dom : $\left(I(X), \tau_{p p}\right) \rightarrow 2^{X}$ is continuous and $\tau_{p p} \subseteq \tau$ (by Theorem 3.3), thus dom is also continuous with respect to $\tau$. Thus $\left.\operatorname{dom}\right|_{J}:(J, \tau) \rightarrow 2^{\mathbb{N}}$ is a continuous bijection and hence an homeomorphism as $J$ is compact Hausdorff. Its inverse is the map $A \mapsto 1_{A}$.

Next result shows that all three topologies $\tau_{1}, \tau_{2}$ and $\tau_{p p}$ are different.

Proposition 3.6. $\quad$ (i) $w_{2}(y)$ is $\tau_{1}$-nowhere dense for all $y \in X$.

(ii) $w_{1}(x)$ is $\tau_{2}$-nowhere dense for all $x \in X$.

Proof. (i) By Theorem 3.2, $\tau_{1}$ is a $T_{1}$ semigroup topology and by Proposition 3.3 each $w_{2}(y)$ is $\tau_{1}$-closed. We show that each $w_{2}(y)$ has empty $\tau_{1}$-interior. Let $V$ be the basic $\tau_{1}$-open set

$$
V=\bigcap_{i=1}^{n} v\left(x_{i}, y_{i}\right) \cap \bigcap_{i=1}^{m} w_{1}\left(z_{i}\right) .
$$

If $y=y_{i}$ for some $i$, then $V \cap w_{2}(y)=\emptyset$. Otherwise, pick $x \notin\left\{x_{1}, \cdots, x_{n}, z_{1}, \cdots, z_{m}\right\}$. Then $V \cap v(x, y) \neq \emptyset$ and $V \cap v(x, y) \cap w_{2}(y)=\emptyset$.

(ii) It is proved analogously to (i).

Proposition 3.7. Let $\tau$ be a semigroup topology on $I(X)$. If $w_{1}(x)$ is open for some $x$, then $w_{1}(y)$ is open for every $y$. Moreover, if $\tau$ is an inverse semigroup topology on $I(X)$ and $w_{1}(x)$ is open for some $x$, then $w_{2}(y)$ is open for every $y$. 
Proof. Let $g \in S_{\infty}(X)$ be such that $g(x)=y$. Let $\varphi: I(X) \rightarrow I(X)$ be given by $\varphi(f)=f \circ g$. Clearly $\varphi$ is a homeomorphism and $w_{1}(y)=\varphi^{-1}\left(w_{1}(x)\right)$. The second claim follows from the first and Lemma 3.1.

The next result is a generalization of the fact that the product topology is the smallest topology with respect to which all projections are continuous.

Theorem 3.8. (i) $\tau_{p p}$ is the smallest topology such that dom : $I(X) \rightarrow 2^{X}$, im : $I(X) \rightarrow 2^{X}$ and $\mathrm{ev}_{x}: D_{x} \rightarrow X(x \in X)$ are continuous, where $2^{X}$ is endowed with the usual product topology and $X$ with the discrete topology.

(ii) dom and $\mathrm{im}$ are open maps when $I(X)$ is endowed with the topology $\tau_{p p}$.

(iii) Let $Y$ be a topological space and $\varphi: Y \rightarrow\left(I(X), \tau_{p p}\right)$ be a map. Then $\varphi$ is continuous if, and only if, dom $\circ \varphi$, im $\circ \varphi$ are continuous and $\mathrm{ev}_{x} \circ \varphi$ is continuous on $\varphi^{-1}\left(D_{x}\right)$ for all $x \in X$.

(iv) The collection of idempotent is $\tau_{p p}$-compact.

Proof. (i) For each $x \in X$, let $v_{x}=\{A \subseteq X: x \in A\}$. Each $v_{x}$ is clopen in $2^{X}$ and they form a subbasis for $2^{X}$. The result follows immediately from the next identities:

$$
w_{1}(x)=(\mathrm{dom})^{-1}\left(2^{X} \backslash v_{x}\right), w_{2}(x)=(\mathrm{im})^{-1}\left(2^{X} \backslash v_{x}\right), \quad v(x, y)=\mathrm{ev}_{x}^{-1}(\{y\}) .
$$

(ii) Let $\left\{x_{i}: 1 \leq i \leq n\right\},\left\{y_{i}: 1 \leq i \leq n\right\},\left\{u_{j}: 1 \leq j \leq m\right\}$ and $\left\{z_{k} 1 \leq k \leq l\right\}$ be finite subsets of $\mathbb{N}$ and consider the basic open set in $I(\mathbb{N})$ :

$$
V=\bigcap_{i=1}^{n} v\left(x_{i}, y_{i}\right) \cap \bigcap_{j=1}^{m} w_{1}\left(u_{j}\right) \cap \bigcap_{k=1}^{l} w_{2}\left(z_{k}\right) .
$$

Then

$$
\{\operatorname{dom}(f): f \in V\}=\left\{A \in 2^{X}: \forall i \leq n\left(x_{i} \in A\right) \text { and } \forall j \leq m\left(u_{j} \notin A\right)\right\} .
$$

is clearly open. Analogously

$$
\{\operatorname{im}(f): f \in V\}=\left\{B \in 2^{X}: \forall i \leq n\left(y_{i} \in B\right) \text { and } \forall k \leq l\left(z_{k} \notin B\right)\right\} .
$$

(iii) Let $\varphi: Y \rightarrow I(X)$ satisfying the hypothesis we will show that $\varphi$ is continuous. Let $V \subseteq I(X)$ be a basic open set as in (ii). Let $y \in \varphi^{-1}(V)$, then $x_{i} \in \operatorname{dom}(\varphi(y))$ for $1 \leq i \leq n$. By (ii) $\operatorname{dom}(V)$ and $\operatorname{im}(V)$ are open in $2^{X}$. We claim that

$$
y \in(\operatorname{dom} \circ \varphi)^{-1}(\operatorname{dom}(V)) \cap(\operatorname{im} \circ \varphi)^{-1}(\operatorname{im}(V)) \cap \bigcap_{i=1}^{n}\left(\operatorname{ev}_{x_{i}} \circ \varphi\right)^{-1}\left(\left\{y_{i}\right\}\right) \subseteq \varphi^{-1}(V) .
$$

In fact, $\operatorname{since} \operatorname{dom}(\varphi(y)) \in \operatorname{dom}(V), u_{i} \notin \operatorname{dom}(\varphi(y))$ for all $j \leq m$. Analogously, $z_{k} \notin \operatorname{im}(\varphi(y))$ for all $k \leq l$. Finally, as $y \in\left(\mathrm{ev}_{x_{i}} \circ \varphi\right)^{-1}\left(\left\{y_{i}\right\}\right)$, then $\varphi(y)\left(x_{i}\right)=y_{i}$. Thus $\varphi(y) \in V$. We have shown that $\varphi^{-1}(V)$ is open and hence $\varphi$ is continuous.

(iv) This follows from Theorem [3.5. In fact, let $\varphi: 2^{X} \rightarrow\left(I(X), \tau_{p p}\right)$ given by $\varphi(A)=1_{A}$. We claim that $\varphi$ is continuous. Notice that $(\operatorname{dom} \circ \varphi)(A)=(\operatorname{im} \circ \varphi)(A)=A$ for all $A$. And $\left(\mathrm{ev}_{x} \circ \varphi\right)(A)=x$ for all $A$ with $x \in A$. Thus, by (iii), $\varphi$ is continuous.

Now we characterize convergence of nets with respect to the topologies defined above.

Theorem 3.9. Let $\left(f_{\lambda}\right)_{\lambda \in \Lambda}$ be a net in $I(X)$ and $f \in I(X)$. Then, $f_{\lambda} \stackrel{\tau_{1}}{\rightarrow} f$ if and only if for all $x \in X$

(i) If $x \in \operatorname{dom}(f)$, then there is $\lambda_{0} \in \Lambda$ such that if $\lambda \geq \lambda_{0}$ then $x \in \operatorname{dom}\left(f_{\lambda}\right)$ and $f_{\lambda}(x)=f(x)$.

(ii) If $x \notin \operatorname{dom}(f)$, then there is $\lambda_{0} \in \Lambda$ such that if $\lambda \geq \lambda_{0}$ then $x \notin \operatorname{dom}\left(f_{\lambda}\right)$. 
Proof. It is straightforward from the definition of $\tau_{1}$.

Theorem 3.10. Let $\left(f_{\lambda}\right)_{\lambda \in \Lambda}$ be a net in $I(X)$ and $f \in I(X)$. Then,

(i) $f_{\lambda} \stackrel{\tau_{1}}{\rightarrow} f$ if and only $f_{\lambda}^{-1} \stackrel{\tau_{2}}{\rightarrow} f^{-1}$.

(ii) $f_{\lambda} \stackrel{\tau_{p p}}{\rightarrow} f$ if and only $f_{\lambda} \stackrel{\tau_{1}}{\rightarrow} f$ and $f_{\lambda} \stackrel{\tau_{2}}{\rightarrow} f$ if, and only if, $f_{\lambda} \stackrel{\tau_{1}}{\rightarrow} f$ and $f_{\lambda}^{-1} \stackrel{\tau_{1}}{\rightarrow} f^{-1}$.

Proof. (i) It follows immediately from the fact that $f \in w_{1}(y)$ iff $f^{-1} \in w_{2}(y)$.

(ii) The first equivalence follows from the fact that $\tau_{p p}$ is the supreme of $\tau_{1}$ and $\tau_{2}$. The second one follows from (i).

\section{The case $X$ Countable}

In this section we will study $I(X)$ when $X$ is a countable set. We will show that $\left(I(\mathbb{N}), \tau_{p p}\right)$ is Polish. We will assume that $X=\mathbb{N}$. In this case $\left(I(\mathbb{N}), \tau_{p p}\right)$ is a Hausdorff, regular and secondcountable space, then by Urysohn theorem, $\left(I(\mathbb{N}), \tau_{p p}\right)$ is metrizable. In order to define a metric compatible with the partial product topology we define some auxiliary functions. Let $f, g \in I(\mathbb{N})$ and consider the function $a_{(f, g)}, b_{(f, g)} \in 2^{\mathbb{N}}$ defined by

$$
\begin{aligned}
& a_{(f, g)}(n)= \begin{cases}0 & \text { if } n \in(\operatorname{dom}(f) \cap \operatorname{dom}(g)) \cup\left((\operatorname{dom}(f))^{c} \cap(\operatorname{dom}(g))^{c}\right), \\
1 & \text { otherwise. }\end{cases} \\
& b_{(f, g)}(n)= \begin{cases}0 & \text { if } n \notin \operatorname{dom}(f) \cap \operatorname{dom}(g), \\
\min \{1,|f(n)-g(n)|\} & \text { if } n \in \operatorname{dom}(f) \cap \operatorname{dom}(g) .\end{cases}
\end{aligned}
$$

Now consider the following metric

$$
\rho(f, g)=\sum_{n \in \mathbb{N}} \frac{a_{(f, g)}(n)+b_{(f, g)}(n)}{2^{n}} .
$$

We show next that $\left(I(\mathbb{N}), \tau_{\rho}\right)$ is a Polish semigroup (but not a topological inverse semigroup). Recall that we also have a metric $\rho^{*}$ on $I(\mathbb{N})$ given by

$$
\rho^{*}(f, g)=\rho\left(f^{-1}, g^{-1}\right) .
$$

Proposition 4.1. $\quad$ (i) $\left(I(\mathbb{N}), \tau_{1}\right)$ is metrizable and $\rho$ is a compatible metric for $\tau_{1}$.

(ii) $\left(I(\mathbb{N}), \tau_{2}\right)$ is metrizable and $\rho^{*}$ is a compatible metric for $\tau_{2}$.

Proof. (i) It is easy to verify that $\rho$ is indeed a metric. Since $\left(I(\mathbb{N}), \tau_{1}\right)$ is metrizable, to show that $\rho$ is compatible with $\tau_{1}$ it suffices to show that they induce the same convergent sequences.

Let $\left(f_{n}\right)_{n \in \mathbb{N}}$ be a sequence and $f \in I(\mathbb{N})$ be such that $f_{n} \stackrel{\rho}{\rightarrow} f$. We use Theorem 3.9 to show that $f_{n} \stackrel{\tau_{1}}{\rightarrow} f$. Let $m \in \mathbb{N}$, there is $N \in \mathbb{N}$ such that if $n \geq N, \rho\left(f_{n}, f\right)<\frac{1}{2^{m}}$, therefore $a_{\left(f, f_{n}\right)}(m)=0$ and $b_{\left(f, f_{n}\right)}(m)=0$, for all $n \geq N$. We consider two cases: (a) Suppose $m \in \operatorname{dom}(f)$. Since $a_{\left(f, f_{n}\right)}(m)=0$ and $b_{\left(f, f_{n}\right)}(m)=0$, for all $n \geq N$, we have that $m \in \operatorname{dom}\left(f_{n}\right)$ and $f_{n}(m)=f(m)$, for all $n \geq N$. (b) Suppose $m \notin \operatorname{dom}(f)$. Since $a_{\left(f, f_{n}\right)}(m)=0$, for all $n \geq N$, we have that $m \notin \operatorname{dom}\left(f_{n}\right)$, for all $n \geq N$. Then, by Theorem 3.9, $f_{n} \stackrel{\tau_{1}}{\rightarrow} f$.

For the other direction, suppose $g_{n} \stackrel{\tau_{1}}{\rightarrow} g$. Let $k \in \mathbb{N}$. Consider the following sets

$$
A=\{m \in \mathbb{N} \mid m \leq k \text { and } m \in \operatorname{dom}(g)\}
$$

and

$$
B=\{m \in \mathbb{N} \mid m \leq \underset{6}{k} \text { and } m \notin \operatorname{dom}(g)\} .
$$


Since $g_{n} \stackrel{\tau_{1}}{\rightarrow} g$, there is $N \in \mathbb{N}$ such that if $n \geq N, g_{n}, g \in v(m, g(m))$, for all $m \in A$ and $g_{n}, g \in w_{1}(m)$, for all $m \in B$. Thus, $a_{\left(g, g_{n}\right)}(m)=0$ and $b_{\left(g, g_{n}\right)}(m)=0$ for all $m \leq k$ and $n \geq N$. Finally, we have that for all $n \geq N$

$$
\rho\left(g, g_{n}\right)=\sum_{t=1}^{\infty} \frac{a_{\left(g, g_{n}\right)}(t)+b_{\left(g, g_{n}\right)}(t)}{2^{t}}=\sum_{t=k+1}^{\infty} \frac{a_{\left(g, g_{n}\right)}(t)+b_{\left(g, g_{n}\right)}(t)}{2^{t}} \leq \sum_{t=k+1}^{\infty} \frac{1}{2^{t}}=\frac{1}{2^{k+1}}<\frac{1}{2^{k}} .
$$

Therefore $g_{n} \stackrel{\rho}{\rightarrow} g$.

(ii) It follows from (i) and Theorem 3.2 .

The following fact shows that $2^{\mathbb{N}}$ is naturally embedded into $I(\mathbb{N})$. Its easy proof is left to the reader.

Proposition 4.2. Let $\eta$ be given by $\eta(A, B)=\rho\left(1_{A}, 1_{B}\right)$, for $A, B \in 2^{\mathbb{N}}$. Then $\eta$ is a compatible metric for $2^{\mathbb{N}}$.

Theorem 4.3. $\quad$ (i) $\left(I(\mathbb{N}), \tau_{1}\right)$ is a Polish semigroup and $\rho$ is a complete compatible metric.

(ii) $\left(I(\mathbb{N}), \tau_{2}\right)$ is a Polish semigroup and $\rho^{*}$ is a complete compatible metric.

Proof. (i) By Proposition 4.1, we only need to show that $\rho$ is complete. Let $\left(f_{n}\right)_{n}$ be a $\rho$-Cauchy sequence. Then $\left(\operatorname{dom}\left(f_{n}\right)\right)_{n}$ is Cauchy in $2^{\mathbb{N}}$ (by Proposition 4.2), so it is convergent. Let $A=$ $\lim _{n \rightarrow \infty} \operatorname{dom}\left(f_{n}\right)$. It is easy to verify that, for each $p \in A,\left(\operatorname{ev}_{p}\left(f_{n}\right)\right)_{n}$ is Cauchy in $\mathbb{N}$ (with the discrete metric) and therefore eventually constant to a value $f(p)$. Thus we have defined a function $f$ such that $\operatorname{dom}(f)=A$ and $f_{n} \stackrel{\rho}{\rightarrow} f$. It is easy to see that $f$ is injective. Finally, from Theorem 3.9 , $f_{n} \stackrel{\tau_{1}}{\rightarrow} f$.

(ii) This follows from (i) and Proposition 2.2.

From the previous result and Proposition 3.6 we conclude that neither $\rho$ nor $\rho^{*}$ are compatible with $\tau_{p p}$. As in Theorem 2.3, we define another metric as follows:

$$
d(f, g)=\rho(f, g)+\rho^{*}(f, g) .
$$

Theorem 4.4. $\left(I(\mathbb{N}), \tau_{p p}\right)$ is a Polish inverse semigroup and $d$ is a complete compatible metric.

Proof. The proof of the compatibility of $d=\rho+\rho^{*}$ with $\tau_{p p}$ is similar to the proof of Theorem 4.1 , The completeness of $d$ follows from Theorem 2.3 and Proposition 4.1 .

\section{OPEN OPERATION}

For $f \in I(X)$, let $r_{f}: I(X) \rightarrow R_{f}$ be given by $r_{f}(g)=g \circ f$ and

$$
R_{f}=\{g \circ f \mid g \in I(X)\} .
$$

Define analogously $l_{f}$ and $L_{f}$ for the corresponding left operation.

In contrast to what happens with topological groups, for a topological semigroup it is not true that $V x$ is open when $V$ is open. Nevertheless, we show that $r_{f}$ is an open map. We need this fact in the next section for proving a uniqueness result for $\tau_{p p}$.

Lemma 5.1. Let $x, y, w, z \in X$. Then

(1) $v(x, y) \circ v(z, x)=v(z, y)$.

(2) $v(x, y) \circ v(z, w)=I(X) \backslash v(z, y)$ if $w \neq x$.

(3) $w_{1}(x) \circ w_{1}(y)=w_{1}(y)$.

(4) $w_{2}(x) \circ w_{2}(y)=w_{2}(x)$. 
(5) $w_{2}(x) \circ w_{1}(y)=w_{2}(x) \cap w_{1}(y)$.

(6) $w_{1}(x) \circ w_{2}(y)=I(X)$.

(7) $w_{1}(y) \circ v(x, y)=w_{1}(x)$.

(8) $w_{1}(z) \circ v(x, y)=I(X)$ if $z \neq y$.

(9) $v(x, y) \circ w_{1}(z)=w_{1}(z)$.

(10) $w_{2}(z) \circ v(x, y)=\left[v(y, x) \circ w_{1}(z)\right]^{-1}=\left[w_{1}(z)\right]^{-1}=w_{2}(z)$.

(11) $v(x, y) \circ w_{2}(z)=\left[w_{1}(z) \circ v(y, x)\right]^{-1}$.

Proof. All items are proved in an analogous way.

1. It's clear that $v(x, y) \circ v(z, x) \subseteq v(z, y)$. For the other inclusion, let $f \in v(z, y)$ and pick $A \subseteq X$ such that $x \in A$ and $|A|=|\operatorname{dom}(f)|$. Let $h: \operatorname{dom}(f) \rightarrow A$ be any bijection such that $h(z)=x$ and let $g=f \circ h^{-1}$. Notice that $g: A \rightarrow \operatorname{im}(f)$ and $g(x)=y$, therefore $h \in v(z, x)$, $g \in v(x, y)$ and $f=g \circ h$.

2. Suppose $w \neq x$. Let $f \in v(x, y) \circ v(z, w)$, then $f=g \circ h$, with $h \in v(z, w)$ and $g \in v(x, y)$. We consider two cases:

(a) Suppose $w \in \operatorname{dom}(g)$. Then $g(w) \neq y$, since $w \neq x$. Notice that $f(z)=g(h(z))=g(w) \neq y$, therefore $f \in I(X) \backslash v(z, y)$.

(b) Suppose $w \notin \operatorname{dom}(g)$. We have that $h(z)=w \notin \operatorname{dom}(g)$, therefore $z \notin \operatorname{dom}(g \circ h)=\operatorname{dom}(f)$. Thus $f \in I(X) \backslash v(z, y)$.

Now let's see that $I(X) \backslash v(z, y) \subseteq v(x, y) \circ v(z, w)$. Let $f \in I(X) \backslash v(z, y)$. We consider four cases.

(a) Suppose $z \notin \operatorname{dom}(f)$ and $y \in \operatorname{im}(f)$. Let $A \subseteq X$ be such that $w, x \in A$ and $|A|=|\operatorname{dom}(f) \cup\{z\}|$. Let $h: \operatorname{dom}(f) \cup\{z\} \rightarrow A$ be any bijection such that $h(z)=w, h\left(f^{-1}(y)\right)=x$ and let $g=f \circ h^{-1}$. Since $h\left(f^{-1}(y)\right)=x$ we have that $g(x)=f\left(h^{-1}(x)\right)=y$, therefore $h \in v(z, x), g \in v(x, y)$ and $f=g \circ h$.

(b) Suppose $z \in \operatorname{dom}(f)$ and $y \notin \operatorname{im}(f)$. Let $A \subseteq X$ be such that $w \in A, x \notin A$ and $|A|=|\operatorname{dom}(f)|$. Let $h: \operatorname{dom}(f) \rightarrow A$ be any bijection such that $h(z)=w$, and let $g: A \cup\{x\} \rightarrow \operatorname{im}(f) \cup\{y\}$ be such that $g(x)=y$ and $g=f \circ h^{-1}$ in $A$. Notice that $h \in v(z, x), g \in v(x, y)$ and $f=g \circ h$.

(c) Suppose $z \in \operatorname{dom}(f)$ and $y \in \operatorname{im}(f)$. Let $A \subseteq X$ be such that $w, x \in A$ and $|A|=|\operatorname{dom}(f)|$. Let $h: \operatorname{dom}(f) \rightarrow A$ be any bijection such that $h(z)=w, h\left(f^{-1}(y)\right)=x$ and let $g=f \circ h^{-1}$. Notice that $g: \operatorname{im}(f) \rightarrow A$ and $g(x)=y$, therefore $h \in v(z, x), g \in v(x, y)$ and $f=g \circ h$.

(d) Suppose $z \notin \operatorname{dom}(f)$ and $y \notin \operatorname{im}(f)$. Let $A \subseteq X$ be such that $w \in A, x \notin A$ and $|A|=$ $|\operatorname{dom}(f) \cup\{z\}|$. Let $h: \operatorname{dom}(f) \cup\{z\} \rightarrow A$ be any bijection such that $h(z)=w$, and let $g: A \cup\{x\} \rightarrow \operatorname{im}(f) \cup\{y\}$ be such that $g(x)=y$ and $g=f \circ h^{-1}$ in $A$. Notice that $h \in v(z, x)$, $g \in v(x, y)$ and $f=g \circ h$.

3. It's obvious that $w_{1}(x) \circ w_{1}(y) \subseteq w_{1}(y)$. For the other inclusion, let $f \in w_{1}(y)$ and pick $A \subseteq X$ such that $x \notin A$ and $|A|=|\operatorname{dom}(f)|$. Let $h: \operatorname{dom}(f) \rightarrow A$ be any bijection and let $g=f \circ h^{-1}$. Notice that $g: \operatorname{im}(f) \rightarrow A$, and therefore $h \in w_{1}(y), g \in w_{1}(x)$ and $f=g \circ h$.

4. It follows from part 3., as $\left[w_{1}(y) \circ w_{1}(x)\right]^{-1}=w_{2}(x) \circ w_{2}(y)$.

5. It's easy to see that $w_{2}(x) \circ w_{1}(y) \subseteq w_{2}(x) \cap w_{1}(y)$. Let $f \in w_{2}(x) \cap w_{1}(y)$. Pick $A \subseteq X$ such that $|A|=|\operatorname{dom}(f)|$. Let $h: \operatorname{dom}(f) \rightarrow A$ be any bijection and let $g=f \circ h^{-1}$. Then $h \in w_{1}(y)$, $g \in w_{2}(x)$ and $f=g \circ h$.

6. Let $f \in I(X)$. Pick $A \subseteq X$ such that $x, y \notin A$ and $|A|=|\operatorname{dom}(f)|$. Let $h: \operatorname{dom}(f) \rightarrow A$ be any bijection and let $g=f \circ h^{-1}$. Then $h \in w_{1}(y), g \in w_{2}(x)$ and $f=g \circ h$.

7. It's easy to see that $w_{1}(y) \circ v(x, y) \subseteq w_{1}(x)$. Let $f \in w_{1}(x)$. Pick $A \subseteq X$ such that $y \notin A$ and $|A|=|\operatorname{dom}(f)|$. Let $h: \operatorname{dom}(f) \cup\{x\} \rightarrow A \cup\{y\}$ be any bijection such that $h(x)=y$, and let $g=f \circ h^{-1}$. Then $h \in v(x, y), g \in w_{1}(y)$ and $f=h \circ g$. 
8. Let $f \in I(X)$. We have two cases.

(a) Suppose that $x \in \operatorname{dom}(f)$. Pick $A \subseteq X$ such that $y \in A, z \notin A$ and $|A|=|\operatorname{dom}(f)|$. Let $h: \operatorname{dom}(f) \rightarrow A$ be any bijection such that $h(x)=y$, and let $g=f \circ h^{-1}$. Then $h \in v(x, y)$, $g \in w_{1}(z)$ and $f=h \circ g$.

(b) Suppose that $x \notin \operatorname{dom}(f)$. Pick $A \subseteq X$ such that $y, z \notin A$ and $|A|=|\operatorname{dom}(f)|$. Let $h: \operatorname{dom}(f)\{x\} \rightarrow A\{y\}$ be any bijection such that $h(x)=y$, and let $g=f \circ h^{-1}$. Then $h \in v(x, y)$, $g \in w_{1}(z)$ and $f=h \circ g$.

9. Let us see that $w_{1}(z) \subseteq v(x, y) \circ w_{1}(z)$. Let $f \in w_{1}(z)$ and suppose that $y \in \operatorname{im}(f)$. Let $A \subseteq X$ be such that $x \in A$ and $|A|=|\operatorname{dom}(f)|$. Let $g: \operatorname{dom}(f) \rightarrow A$ be any bijection such that $g\left(f^{-1}(y)\right)=x$ and let $h=f \circ g^{-1}$. Then $f=h \circ g, h \in v(x, y)$.

Now, suppose that $f \in w_{1}(z)$ and $y \notin \operatorname{im}(f)$. Let $A \subseteq X$ be such that $x \notin A$ and $|A|=|\operatorname{dom}(f)|$. Let $g: \operatorname{dom}(f) \rightarrow A$ be any bijective function. Let $h: A \cup\{x\} \rightarrow \operatorname{im}(f) \cup\{y\}$ be such that $h(x)=y$ and $(h \circ g)(v)=f(v)$ for $v \in \operatorname{dom}(f)$. We have that $h \in v(x, y), g \in w_{1}(z)$ and $f=h \circ g$, that is, $f \in v(x, y) \circ w_{1}(z)$.

Finally, 10. and 11. are evident.

Lemma 5.2. Let $A, B, C \subseteq I(X)$, then $A \circ(B \cap C) \subseteq(A \circ B) \cap(A \circ C)$.

Proof. Straightforward.

Now we can show that $r_{f}$ is an open map.

Theorem 5.3. Let $f \in I(X)$. Then, $r_{f}: I(X) \rightarrow R_{f}$ is an open map where $R_{f}$ is endowed with the relative topology to $\tau_{p p}$

Proof. Let $U$ be a non empty basic $\tau_{p p}$-open set of the form:

$$
U=\bigcap_{i=1}^{n} v\left(x_{i}, y_{i}\right) \cap \bigcap_{i=1}^{m} w_{1}\left(z_{i}\right) \cap \bigcap_{i=1}^{p} w_{2}\left(w_{i}\right)
$$

We will show that $U \circ f$ is open. Consider the sets:

$$
\begin{aligned}
\widehat{M} & =\left\{x_{i}: 1 \leq i \leq n\right\} & M & =\widehat{M} \cap \operatorname{im}(f) \\
\widehat{N} & =\left\{z_{i}: 1 \leq i \leq m\right\} & N & =\widehat{N} \cap \operatorname{im}(f) \\
\widehat{O} & =\left\{w_{i}: 1 \leq i \leq p\right\} & O & =\widehat{O} \cap \operatorname{im}(f)
\end{aligned}
$$

and

$$
Q=\bigcap_{x_{i} \in M} v\left(f^{-1}\left(x_{i}\right), y_{i}\right) \cap \bigcap_{z_{i} \in N} w_{1}\left(f^{-1}\left(z_{i}\right)\right) \cap \bigcap_{x_{i} \in \widehat{M} \backslash M} w_{2}\left(y_{i}\right) \cap \bigcap_{i=1}^{p} w_{2}\left(w_{i}\right) .
$$

We claim that $U \circ f=Q \cap R_{f}$. First, we show that $U \circ f \subseteq Q$. Let

$$
\begin{aligned}
R & =\bigcap_{x_{i} \in M} v\left(f^{-1}\left(x_{i}\right), x_{i}\right) \cap \bigcap_{z_{i} \in N} v\left(f^{-1}\left(z_{i}\right), z_{i}\right) \cap \bigcap_{w_{i} \in O} v\left(f^{-1}\left(w_{i}\right), w_{i}\right) \\
S & =\bigcap_{x_{i} \in \widehat{M} \backslash M} w_{2}\left(x_{i}\right) \cap \bigcap_{x_{i} \in \widehat{N} \backslash N} w_{2}\left(z_{i}\right) \cap \bigcap_{x_{i} \in \widehat{O} \backslash O} w_{2}\left(w_{i}\right) .
\end{aligned}
$$

Then $f \in R \cap S$. By Lemma 5.1, we have the following:

- $v\left(x_{i}, y_{i}\right) \circ v\left(f^{-1}\left(x_{i}\right), x_{i}\right)=v\left(f^{-1}\left(x_{i}\right), y_{i}\right)$, for all $x_{i} \in M$.

- $w_{1}\left(z_{i}\right) \circ v\left(f^{-1}\left(z_{i}\right), z_{i}\right)=w_{1}\left(f^{-1}\left(z_{i}\right)\right)$, for all $z_{i} \in N$.

- $w_{2}\left(w_{i}\right) \circ v\left(f^{-1}\left(w_{i}\right), w_{i}\right)=w_{2}\left(w_{i}\right)$, for all $w_{i} \in O$. 
- $v\left(x_{i}, y_{i}\right) \circ w_{2}\left(x_{i}\right)=w_{2}\left(y_{i}\right)$, for all $x_{i} \in \widehat{M} \backslash M$.

- $w_{1}\left(z_{i}\right) \circ w_{2}\left(z_{i}\right)=I(X)$, for all $z_{i} \in \widehat{N} \backslash N$.

- $w_{1}\left(w_{i}\right) \circ w_{2}\left(w_{i}\right)=w_{2}\left(w_{i}\right)$, for all $w_{i} \in \widehat{O} \backslash O$.

Therefore, using Lemma 5.2 and the claims above, we easily have that

$$
\begin{aligned}
U \circ f & \subseteq U \circ(R \cap S) \\
& \subseteq \bigcap_{x_{i} \in M} v\left(f^{-1}\left(x_{i}\right), y_{i}\right) \cap \bigcap_{z_{i} \in N} w_{1}\left(f^{-1}\left(z_{i}\right)\right) \cap \bigcap_{w_{i} \in O} w_{2}\left(w_{i}\right) \cap \bigcap_{x_{i} \in \widehat{M} \backslash M} w_{2}\left(y_{i}\right) \cap \bigcap_{w_{i} \in \widehat{O} \backslash O} w_{2}\left(w_{i}\right) \\
& =\bigcap_{x_{i} \in M} v\left(f^{-1}\left(x_{i}\right), y_{i}\right) \cap \bigcap_{x_{i} \in N} w_{1}\left(f^{-1}\left(z_{i}\right)\right) \cap \bigcap_{i=1}^{p} w_{2}\left(w_{i}\right) \cap \bigcap_{x_{i} \in \widehat{M} \backslash M} w_{2}\left(y_{i}\right) \\
& =Q .
\end{aligned}
$$

Now, we are going to show that $Q \cap R_{f} \subseteq U \circ f$. Let $h \in Q \cap R_{f}$ and define $g=h \circ f^{-1}$. Notice that $g\left(x_{i}\right)=y_{i}$, for all $x_{i} \in M$, since $h \in v\left(f^{-1}\left(x_{i}\right), y_{i}\right)$ for $x_{i} \in M$. Notice also that that if $x_{i} \in \widehat{M} \backslash M$, then $x_{i} \notin \operatorname{dom}(g)$, and $y_{i} \notin \operatorname{im}(g)$ since $h \in w_{2}\left(y_{i}\right)$ for $x_{i} \in \widehat{M} \backslash M$. Therefore we can extend $g$ to a function $\widehat{g}$ such that $\operatorname{dom}(\widehat{g})$ is $\operatorname{dom}(g) \cup(\widehat{M} \backslash M)$ and $g\left(x_{i}\right)=y_{i}$, for all $x_{i} \in \widehat{M} \backslash M$.

To finish the proof it suffices to show that $h=\widehat{g} \circ f$ and $\widehat{g} \in U$. First, we show that $\widehat{g} \in U$.

- $\widehat{g}\left(x_{i}\right)=y_{i}$, for all $i$, by construction.

- $z_{i} \notin \operatorname{dom}(\widehat{g})$, for all $z_{i}$. In fact, if $z_{i} \in \widehat{N} \backslash N$, then $z_{i} \notin \operatorname{im}(f)$, therefore $z_{i} \notin \operatorname{dom}(g)$ and thus $z_{i} \notin \operatorname{dom}(\widehat{g})$. On the other hand, if $z_{i} \in N$, then $h \in w_{1}\left(f^{-1}\left(z_{i}\right)\right)$ and $z_{i} \notin \operatorname{dom}(\widehat{g})$.

- As $h \in w_{2}\left(w_{i}\right)$ for all $w_{i}$, we have that $w_{i} \notin \operatorname{im}(\widehat{g})$, for all $w_{i}$.

Thus $\widehat{g} \in U$. Finally, since $g=h \circ f^{-1}$ and $\operatorname{dom}(\widehat{g}) \cap \operatorname{im}(f)=\operatorname{dom}(g), h=\widehat{g} \circ f$.

Theorem 5.4. Let $f \in I(X)$ and consider $L_{f}=\{f \circ g \mid g \in I(X)\}$. Then, $\circ_{f}: I(X) \rightarrow L_{f}$, such that $\circ_{f}(g)=f \circ g$, is an open function, where $L_{f}$ is endowed with the relative topology to $\tau_{p p}$.

Proof. Similar the proof of Theorem 5.3.

We conjecture that $\circ$ is an open map. To show it would require analyzing many cases in order to extend Lemma 5.1. We did not pursue it further, since we do not need this result for this paper.

\section{UNIQUENESS OF $\tau_{p p}$}

In this section we present some results showing that $\tau_{p p}$ is unique in some sense. For that end we first show that $\left(I(X), \tau_{p p}\right)$ is a quotient of a symmetric group $S_{\infty}(Y)$ (for some $Y$ ) with the usual product topology.

Let $X \subseteq Y$. For each $f \in S_{\infty}(Y)$ we define $\widehat{f} \in I(X)$ as follows:

$$
\widehat{f}=\{(x, f(x)): x \in X \text { and } f(x) \in X\} .
$$

Thus $\widehat{f}$ is the restriction of $f$ to $f^{-1}(X) \cap X$. Let $\pi: S_{\infty}(Y) \rightarrow I(X)$ be given by $\pi(f)=\widehat{f}$.

Proposition 6.1. Let $X$ be an infinite set and $X \subseteq Y$. Then $\pi$ is onto if, and only if, $|X| \leq|Y \backslash X|$.

Proof. Suppose $|X| \leq|Y \backslash X|$ and let $g \in I(X)$. Let $A=\operatorname{dom}(g)$ and $B=\operatorname{im}(g)$. Let $C \subseteq Y \backslash X$ and $D \subseteq Y \backslash X$ be such that $|C|=|X \backslash A|,|D|=|X \backslash B|,|Y \backslash(X \cup C)|=|Y \backslash(X \cup D)|=|Y|$. All this conditions can be fulfilled as $|X| \leq|Y \backslash X|$. Now take any extension of $g$ to a bijection $f: Y \rightarrow Y$ such that $f[X \backslash A]=C, f[D]=X \backslash B$. Then $\widehat{f}=g$. 
Conversely, suppose $|X|>|Y \backslash X|$. Let $X=A \cup B$ be a partition of $X$ into sets of equal cardinality. Then there is no $f \in S_{\infty}(Y)$ such that $\widehat{f}=1_{A}$.

Let us observe that the map $\pi$ is not a semigroup homomorphism. In fact, let $y \in Y \backslash X, x \in X$, and $f \in S_{\infty}(Y)$ be such that $f(x)=y, f(y)=x$ and $f(z)=z$ for all $z \notin\{x, y\}$. Then $f \circ f=1_{Y}$, $\pi\left(1_{Y}\right)=1_{X}$ but $\pi(f) \circ \pi(f)=1_{X \backslash\{x\}}$. In general, $\pi(f) \circ \pi(g) \subseteq \pi(f \circ g)$.

Proposition 6.2. Let $X \subseteq Y$ with $|X| \leq|Y \backslash X|$. The map $\pi: S_{\infty}(Y) \rightarrow\left(I(X), \tau_{p p}\right)$ is continuous, onto and open.

Proof. First, we will show that $\pi$ is continuous. Let $u(x, y)$ denote the subbasic open set of $S_{\infty}(Y)$ given by $\left\{f \in S_{\infty}(Y): f(x)=y\right\}$ for $x, y \in Y$. The continuity of $\pi$ follows from the following identities.

(i) $\pi^{-1}(v(x, y))=u(x, y)$ for all $x, y \in X$.

(ii) $\pi^{-1}\left(w_{1}(x)\right)=\bigcup_{y \in Y \backslash X} u(x, y)$ for $x \in X$.

(iii) $\pi^{-1}\left(w_{2}(y)\right)=\bigcup_{x \in Y \backslash X} u(x, y)$ for $y \in X$.

To see that $\pi$ is open, let $x_{i}, y_{i}$ in $Y$ for $1 \leq i \leq n$. Let $1 \leq k_{1} \leq k_{2} \leq k_{3} \leq n$ be such that (1) $x_{i}, y_{i} \in X$ for $1 \leq i \leq k_{1}$, (2) $x_{j} \in X$ and $y_{j} \notin X$ for $k_{1}<j \leq k_{2}$, (3) $x_{j} \notin X$ and $y_{j} \in X$ for $k_{2}<j \leq k_{3}$ and (4) $x_{j} \notin X$ and $y_{j} \notin X$ for $k_{3}<j \leq n$. Then

$$
\pi\left(\bigcap_{i=1}^{n} u\left(x_{i}, y_{i}\right)\right)=\bigcap_{i=1}^{k_{1}} v\left(x_{i}, y_{i}\right) \cap \bigcap_{j=k_{1}+1}^{k_{2}} w_{1}\left(x_{j}\right) \cap \bigcap_{j=k_{2}+1}^{k_{3}} w_{2}\left(y_{j}\right) .
$$

The direction $\subseteq$ is straightforward. For $\supseteq$ we use an analogous construction as in the proof that $\pi$ is onto (Proposition 6.1).

Theorem 6.3. Let $X \subseteq Y$ with $|Y \backslash X| \geq|X|$. $\tau_{p p}$ is the only inverse semigroup Hausdorff topology on $I(X)$ with respect to which $\pi: S_{\infty}(Y) \rightarrow I(X)$ is continuous.

Proof. Let $\tau$ be an inverse semigroup Hausdorff topology on $I(X)$ such that $\pi$ is continuous. Then by Theorem 3.3 we have that $\tau_{p p} \subseteq \tau$. Since $\pi$ is continuous, by Proposition 6.2 we conclude that $\tau \subseteq \tau_{p p}$.

We now present a proof that $\tau_{p p}$ is the unique inverse semigroup Polish topology on $I(\mathbb{N})$ (satisfying some addional conditions). Our approach is different than the one used in [2]. We need two auxiliary results.

Lemma 6.4. Let $R_{A}=\left\{f \circ 1_{A}: f \in I(X)\right\}$ and $r_{A}: I(X) \rightarrow R_{A}, r_{A}(f)=f \circ 1_{A}$. Let $\tau$ be an inverse semigroup Hausdorff topology on $I(X)$. Suppose $r_{A}$ is a $\tau$-open map for every $A \subseteq X$ cofinite. Let $\left(f_{k}\right)_{k}$ be a sequence on $I(X)$ such that

(i) $f_{k} \stackrel{\tau_{p p}}{\longrightarrow} f$.

(ii) There is a cofinite set $A \subseteq X$ such that $f_{k} \circ 1_{A} \stackrel{\tau}{\longrightarrow} f \circ 1_{A}$.

Then $f_{k} \stackrel{\tau}{\longrightarrow} f$.

Proof. Let $A$ be as in (ii). Let $V \in \tau$ with $f \in V$. Since $\tau_{p p} \subseteq \tau$ (by Theorem 3.3), we can assume that $V \subseteq v(x, f(x)) \cap w_{1}(z)$ for all $x \in \operatorname{dom}(f) \cap(X \backslash A)$ and all $z \in(X \backslash A) \backslash \operatorname{dom}(f)$. By hypothesis, $V \circ 1_{A}$ is open in $R_{A}$. By (ii), there is $k_{0}$ such that $f_{k} \circ 1_{A} \in V \circ 1_{A}$ for all $k \geq k_{0}$. Thus there is $g_{k} \in V$ such that $f_{k} \circ 1_{A}=g_{k} \circ 1_{A}$ for all $k \geq k_{0}$. By (i) and theorems 3.8 and 3.9, there is $k_{1} \geq k_{0}$ such that $\operatorname{dom}\left(f_{k}\right) \cap(X \backslash A)=\operatorname{dom}(f) \cap(X \backslash A)$ and $f_{k}(x)=f(x)$ for all $x \in \operatorname{dom}(f) \cap(X \backslash A)$ 
and all $k \geq k_{1}$. Since $g_{k} \in V$, $\operatorname{dom}\left(g_{k}\right) \cap(X \backslash A)=\operatorname{dom}(f) \cap(X \backslash A)$ and $g_{k}(x)=f(x)$ for all $x \in \operatorname{dom}(f) \cap(X \backslash A)$. As $f_{k} \circ 1_{A}=g_{k} \circ 1_{A}$, then $f_{k}=g_{k}$ and hence $f_{k} \in V$ for all $k \geq k_{1}$.

Lemma 6.5. Let $\tau$ be a Polish inverse semigroup topology on $I(\mathbb{N})$ such that $A \mapsto 1_{A}$ from $2^{\mathbb{N}}$ to $(I(\mathbb{N}), \tau)$ is continuous. Let $\mathbb{N} \subseteq Y$ with $Y \backslash \mathbb{N}$ infinite and $f_{k} \in S_{\infty}(Y), k \in \mathbb{N}$ such that $f_{k} \rightarrow f$. There is a dense $G_{\delta}$ set $G \subseteq S_{\infty}(Y)$ such that

$$
\widehat{f_{k}} \circ \widehat{g} \stackrel{\tau}{\longrightarrow} \widehat{f} \circ \widehat{g} \text { for all } g \in G \text {. }
$$

Proof. By Theorem 3.3 we have that $\tau_{p p} \subseteq \tau$. Since both topologies are Polish, by a well know classical result, they have the same Borel sets (see [5, Exercise 15.4]). As $\pi$ is continuous with respect to $\tau_{p p}$, we have that $\pi: S_{\infty}(Y) \rightarrow(I(\mathbb{N}), \tau)$ is Borel measurable. Thus, there is a dense $G_{\delta}$ set $H \subseteq S_{\infty}(Y)$ such that $\left.\pi\right|_{H}: H \rightarrow(I(\mathbb{N}), \tau)$ is continuous (see [5, Theorem 8.38]). Let

$$
L_{k+1}=\left\{g \in S_{\infty}(Y): f_{k} \circ g \in H\right\}, \quad L_{0}=\left\{g \in S_{\infty}(Y): f \circ g \in H\right\} .
$$

Let $G=\bigcap_{k} L_{k}$. Since each $L_{k}$ is dense $G_{\delta}$, so is $G$. As $f_{k} \circ g \rightarrow f \circ g$ (in $S_{\infty}(Y)$ ) for all $g \in G$ and $\pi$ is continuous in $H, \pi\left(f_{k} \circ g\right) \stackrel{\tau}{\longrightarrow} \pi(f \circ g)$ for all $g \in G$.

As we said before, $\pi$ is not a homomorphism, however, we have the following

$$
\pi\left(f_{k}\right) \circ \pi(g)=\pi\left(f_{k} \circ g\right) \circ 1_{\operatorname{dom}\left(\pi\left(f_{k}\right) \circ \pi(g)\right)} .
$$

Notice that $\pi\left(f_{k}\right) \stackrel{\tau_{p p}}{\longrightarrow} \pi(f)$ as $\pi$ is continuous with respect to $\tau_{p p}$ (by Proposition 6.2). As the function dom is continuous (by Theorem 3.8), we have

$$
\operatorname{dom}\left(\pi\left(f_{k}\right) \circ \pi(g)\right) \rightarrow \operatorname{dom}(\pi(f) \circ \pi(g))
$$

where the convergence is in the Cantor space $2^{\mathbb{N}}$. Finally, by hypothesis the map $A \mapsto 1_{A}$ is continuous with respect to $\tau$, thus we conclude

$$
\pi\left(f_{k}\right) \circ \pi(g) \stackrel{\tau}{\longrightarrow} \pi(f) \circ \pi(g)
$$

for all $g \in G$.

Theorem 6.6. $\tau_{p p}$ is the unique inverse semigroup Polish topology on $I(\mathbb{N})$ such that the collection of idempotent is compact and $r_{A}$ is a $\tau$-open map for every $A \subseteq \mathbb{N}$.

Proof. By Theorem 5.1, $r_{A}$ is a $\tau_{p p}$-open map and, by Theorem 3.8, the collection of idempotent is $\tau_{p p}$-compact. Conversely, let $\tau$ be a topology on $I(\mathbb{N})$ as in the hypothesis. Let $\mathbb{N} \subseteq Y$ be such that $Y \backslash \mathbb{N}$ is countable. To have that $\tau=\tau_{p p}$, it suffices to show, by Lemma 6.5, that $\pi: S_{\infty}(Y) \rightarrow(I(\mathbb{N}), \tau)$ is continuous.

Let $f_{k} \in S_{\infty}(Y)$ be a sequence converging to $f$. Let $G \subseteq S_{\infty}(Y)$ be a dense $G_{\delta}$ as in Proposition 6.5. Since $\left\{h \in S_{\infty}(Y): \operatorname{im}(\widehat{h})\right.$ is cofinite $\}$ is dense $G_{\delta}$, let $g \in G$ be such that $\operatorname{im}(\widehat{g})$ is cofinite. Now notice that $\widehat{g} \circ \widehat{g}^{-1}=1_{\mathrm{im}(\widehat{g})}$. Let $A=\operatorname{im}(\widehat{g})$. Since $\widehat{f}_{k} \circ \widehat{g} \stackrel{\tau}{\longrightarrow} \widehat{f} \circ \widehat{g}$, by the continuity of $\circ$ we have $\widehat{f}_{k} \circ 1_{A} \stackrel{\tau}{\longrightarrow} \widehat{f} \circ 1_{A}$. Thus by Lemma 6.4, we conclude that $\widehat{f}_{k} \stackrel{\tau}{\longrightarrow} \widehat{f}$.

\section{REFERENCES}

[1] Elliott, L., Jonušas, J., Mitchell, J. D., Morayne, M. and Péresse, Y. Automatic continuity and uniqueness of Polish semigroup topologies. arXiv:1912.07029, 2019.

[2] Elliott, L., Jonušas, J., Mesyan, Z., Mitchell, J. D., Morayne, M. and Péresse, Y. Automatic continuity, unique Polish topologies, and Zariski topologies (Part I, monoids). arxiv.org/abs/1912.07029v3 2020.

[3] Gao, S. Invariant Descriptive Set Theory, Chapmann \& Hall, 2009.

[4] Kallman, R., A uniqueness result for the infinite symmetric group. In Studies in analysis, volume 4 of Adv. in Math. Suppl. Stud., pages 321-322. Academic Press, New York-London, 1979.

[5] Kechris, A. Classical Descriptive Set Theory. Graduate Texts in Mathematics 156. Springer-Verlag, New York, 1995.

[6] Mesyan, Z., Mitchell, J. and Péresse, Y. Topological transformation monoids. arXiv:1809.04590, 2018. 
[7] Pérez, J. Topologías sobre semigrupos inversos. Tesis de maestría. Universidad Industrial de Santander, December 2019.

[8] Rosendal, C. and Solecki, S. Automatic continuity of homomorphisms and fixed points on metric compacta. Israel J. Math., 162:349-371, 2007.

Escuela de Matemáticas, Universidad Industrial de Santander, C.P. 680001, Bucaramanga - ColomBIA.

Email address: jersonenrique_64@hotmail.com

Escuela de Matemáticas, Universidad Industrial de Santander, C.P. 680001, Bucaramanga - ColomBIA.

Email address: cuzcatea@saber.uis.edu.co 